\title{
Management of intra-operative tracheal injuries during transhiatal esophagectomy
}

\author{
Farooq Ahmad Ganie*, Ghulam Nabi Lone, Syed Mohsin Manzoor, Hakeem Zubair Ashraf, \\ Nadeem-ul Nazir Kawoosa, Rouf Gul
}

Department of Cardiovascular and Thoracic Surgery, Sheri-I-Kashmir Institute of

Medical Sciences (SKIMS), Soura, Srinagar, Kashmir, India

\begin{abstract}
Background and objective: In transhiatal esophagectomy, iatrogenic injuries to trachea are very uncommon but when it happens it is potentially lethal and has high morbidity. This study aimed to investigate the incidence and outcome of tracheal injuries during transhiatal esophagectomy.

Methods: The medical records of 608 patients who underwent transhiatal esophagectomy for esophageal cancer from January 2000 to January 2019 were analyzed.

Results: Out of 608 transhiatal esophagectomy, four $(0.66 \%)$ patients sustained injuries to major airway. Three injuries occurred during transhiatal and one injury during transcervical part of dissection. All the injuries occurred in trachea proximal to carina. All four injuries were closed primarily, re-enforced by muscle and fascial pledgets.

Conclusion: Tracheobronchial injury is a rare complication of transhiatal esophagectomy, mostly seen in patients who receive neo-adjuvant therapy or have locally advanced growth with dense adhesions. Its immediate recognition and closure decreases the mortality and morbidity associated with this rare but fatal intra-operative complication. It can be managed effectively by primary closure, with or without muscle and fascial pledget reinforcement.
\end{abstract}

IMC J Med Sci 2021; 15(1): 003

\section{Introduction}

The squamous cell cancer of esophagus is common in Asian countries of esophageal cancer belt, stretching from eastern Turkey to northern China and India. It is relatively uncommon in United States, Canada and Europe where adenocarcinoma of the lower esophagus and cardia predominate [1]. The predominant risk factors for squamous cell carcinoma are smoking and alcohol consumption and for adenocarcinoma it is gastro-esophageal reflux and Barrett's esophagus [2]. The most common surgical procedure performed for esophageal cancers is transhiatal esophagectomy. Other options are Ivor-Lewis, McKeown procedure and pharyngo-laryngo-esophagectomy (PLE) for hypopharyngeal and upper cervical esophageal lesions. The rationale for transhiatal esophagectomy is to avoid thoracotomy and its complications. Fashioning of cervical anastomosis is to minimize clinical consequences of anastomotic leak. The common complications include anastomotic leak/stricture, recurrent laryngeal nerve injury, bleeding, and chyle leak. There is a risk of injury to azygous vein, trachea and cardiac instability. Injury to major airway is a rare but potentially fatal complication of transhiatal esophagectomy that needs prompt recognition, isolation and repair [3]. This study aimed to investigate the incidence and outcome of tracheal injuries during transhiatal esophagectomy.

*Correspondence: Farooq Ahmad Gganie, Department of Cardiovascular and Thoracic Surgery, Sheri-I-Kashmir Institute of Medical Sciences (SKIMS), Soura, Srinagar, Kashmir, India. Email ID: farooq.ganie@ymail.com 
Materials and methods

Study place and population: This retrospective study was conducted in the Department of Cardiovascular and Thoracic surgery, Sheri-IKashmir Institute of Medical Sciences, Srinagar, Kashmir, India. The medical records of 750 patients operated for esophageal cancer from January 2000 to January 2019 were analyzed. Out of 750 patients, 608 patients underwent transhiatal esophagectomy and 142 had trans-thoracic resection. Patients who underwent transhiatal esophagectomy were included in the study. Patients with early stages of disease did not receive neo-adjuvant therapy while patients with advanced growth (stage IIb and III) received neo-adjuvant chemo-radiotherapy before surgery. Records of complete history, physical examination, complete blood count, biochemical profile, electrocardiography, endoscopic biopsy, barium esophagogram, ultrasound abdomen and computed tomography of chest and abdomen, surgical procedures, injury recognition and immediate management, approach and technique of repair and postoperative cares of all included cases were evaluated.

Surgical procedure: Supra-umbilical midline laparotomy was performed, tumor resectability was assessed, gastric mobilization was performed and esophageal hiatus was enlarged for exposure of intra-thoracic esophagus. Lymph node dissection in lower mediastinum around esophago-gastric junction, lesser curvature of stomach and celiac axis was performed. Thoracic esophagus was mobilized by blunt finger dissection via esophageal hiatus and cervical incision such that the two hands met each other in the posterior mediastinum while mobilizing the esophagus and freeing it from surrounding structures. Gastrointestinal continuity re-established by esophago-gastric anastomosis fashioned in the neck through cervical incision. Chest drains were inserted if pleurae were breached and a feeding jejunostomy was placed routinely in all patients. Four patients sustained tracheal injury, three during transhiatal and one during trans-cervical part of dissection. The laceration ranged from 3.5 to $>5 \mathrm{~cm}$ in length. The operative repair of tracheal injury was completed in $10-30 \mathrm{~min}$. Two patients needed right postero- lateral thoracotomy and two patients needed extended cervical incision to repair their tracheal tear.

Injury recognition and immediate management: Injury to trachea occurred during mediastinal phase of esophageal dissection. The tracheal injuries were recognized immediately due to loss of airway resistance during ventilation and gush of air leaking through the incision site. The rent in the trachea was assessed by surgeon's finger and/or direct visualization. The endotracheal tube was advanced into the right bronchus distal to the injured area to establish adequate ventilation and the tear area was packed with moist gauze pack till patient's ventilation was stable and acceptable for repair.

Approach and technique of repair: Tracheal tear was repaired by trans-cervical approach in two and trans-thoracic approach in two patients. In transcervical approach, longitudinal incision along the anterior border of sternocleidomastoid on left side was extended up to the superior border of manubrium. The neck was hyper-extended and rotated to right. The surgeon positioned on the head end, trachea was retracted medially and anteriorly, sternocleidomastoid laterally and manubrium sternum lifted anteriorly. The cervical esophageal stump was retracted cranially to widen retro-tracheal space.

The tracheal rent was sutured with interrupted polypropylene (4.0) suture using long instruments from distal to proximal. The sutures were buttressed with muscle and fascial pledgets. Care was taken not to puncture the endotracheal tube. At the end of tracheal repair the endotracheal tube was withdrawn to ensure there was no inadvertent suturing of the airway tube. The gastric tube was advanced into the neck, anastomosed to esophageal stump and the procedure completed. Trans-thoracic approach was used in patients with longer tear $(>5 \mathrm{~cm})$ in the trachea. Right posterolateral thoracotomy through $5^{\text {th }}$ intercostals space was performed. The trachea was dissected from surrounding structures and the tear was sutured with interrupted $\left(4^{0}\right)$ polypropylene sutures and then the gastric tube was advanced to neck for anastomosis with the proximal esophageal stump. Thoracotomy was closed after placing one intercostal tube drain (32 F) in the pleural cavity. 
Postoperative care: No patient needed postoperative ventilation. Breathing exercises were encouraged. One patient developed bronchopneumonia, however, responded to antibiotics and supportive measures. Feeding was started on $2^{\text {nd }}$ post-operative day. Integrity of esophageal anastomosis was checked on $7^{\text {th }}$ postoperative day.

\section{Results}

Out of 608 transhiatal esophagectomies, four patients $(0.66 \%)$ sustained injuries to major airway. Three injuries occurred during transhiatal and one injury during transcervical part of dissection. All the injuries occurred in trachea proximal to carina. All four injuries were closed primarily, re-enforced by muscle and fascial pledgets. In two patients the trachea was repaired through right thoracotomy and in two patients by the cervical incision that was utilized for mobilization of esophagus in the neck and provided adequate exposure for repair of the trachea. Two patients who sustained intraoperative airway injury received pre-operative chemo-radiation for advanced growth.

\section{Discussion}

Transhiatal esophagectomy has been the favored operative approach in our institution for carcinoma of esophagus below carina and type I and II tumors of esophagogastric junction. Reconstruction is usually performed by iso-peristaltic gastric tube which functions as neo-esophagus after surgery. In patients who need esophagectomy along with total gastrectomy, jejunal or colonic interposition is mandatory. Other treatment options include neoadjuvant or adjuvant chemo-radiotherapy. Advocates of trans-thoracic approach argue that it reduces rates of circumferential resection margin (CRM) involvement and risks loco-regional recurrence. However, the mediastinal lymph node involvement reflects systemic micro metastasis and extended resection does not alter natural course of disease. Regardless of the operative technique, it is often difficult to obtain circumferential clearance due to proximity of vital structures and lack of fascial boundaries. Neo-adjuvant therapies have improved surgical and oncological outcomes. It is proven that transhiatal esophagectomy is a safe approach with advantages in post-operative recovery and oncological outcomes have improved when combined with neo-adjuvant therapy. Tracheal injury is a rare, dreaded and potentially fatal complication of esophagectomy [4-6], which should be managed immediately and meticulously to decrease intra-operative mortality and postoperative morbidity and mortality. Management of major airway injury during transhiatal esophagectomy is challenging as there is no direct access to membranous trachea during the procedure. This invariably entails the performance of thoracotomy or an anterior sternal split in order to facilitate repair, which adds to the morbidity $[5,7]$. The incidence of tracheal injury was found to be similar in two large reports comparing the transhiatal with open thoracic approach [4,5]. However, Hulscher et al. showed that airway injury is higher in transhiatal than trans-thoracic esophagectomy [7]. In our study the incidence of tracheal injury during transhiatal esophagectomy was $0.66 \%$. The incidence of air way injury reported in the published literature is $0.4 \%$ and $0.6 \%[8,9]$. In our study, primary repair of air way injury was carried out by suturing with interrupted polypropylene suture material with fascial and muscle pledget augmentation. Primary suturing of tracheal injuries during transhiatal esophagectomy has also been reported by other authors $[4,5]$. A variety of technical modifications to avoid thoracotomy have been described to manage intraoperative tracheal injury. Use of transposed stomach to patch the laceration is suitable for small tears [4]. Use of pericardial patch reinforcement of the repair with PTFE has been reported [10]. Augmentation of primary repair with gastric tube was associated with pulmonary complications, leading to prolonged assisted ventilation and prolonged stay in the intensive care unit, but mortality was rare [5]. We used trans-cervical approach in two patients who had proximal airway tear by anterior sternal lift, with no airway injury related morbidity and mortality. The same approach has been used by Gupta et al. in patients with tracheal injuries during transhiatal esophagectomy with good results [6]. Harney et al. described the successful use of laparoscopic instruments to repair intra-operative tracheal injury 
extending up to the carina [3]. Two patients who sustained intra-operative airway injury had received pre-operative chemo-radiation for advanced growth. Suryanarayana et al. inferred from his study that direct tumor infiltration, radiotherapy, peri-tumor malignant inflammation or infection and abscess formation, all increase the risk of injury to the peri-esophageal structures [11]. Radiotherapy weakens the tracheal wall making dissection difficult and trachea vulnerable to damage during blunt dissection [12]. There are many reports and studies of airway injuries during esophagectomy, some with a fatal outcome $[8,9,11,13]$. In our series, there was no mortality related to airway injury. Two patients in our series with dense adhesions and tracheal injury $>5 \mathrm{~cm}$ needed thoracotomy to repair the airway injury. In case of suspected airway injury early conversion to trans-thoracic approach is a better option as identification of extent and repair of tracheal injury is technically easy and rapid, till then packing the leak helps to gain time for conversion. We did not use double lumen endotracheal tube during transhiatal esophagectomy for ventilation; therefore single lumen endotracheal tube was advanced into main bronchus for ventilation in patients who sustained airway injury. Although lung isolation is not necessary in transhiatal esophagectomy, yet cases with tumors in close proximity to the major airway or patients who have undergone radiotherapy may benefit from an electively positioned double lumen endotracheal tube [13].

In patients with carcinoma esophagus having dense peri-esophageal adhesions after neo-adjuvant chemo-radiation and difficult transhiatal esophageal dissection due to adherent growth, threshold for conversion to trans-thoracic approach (and sharp dissection under vision) should be low. Injury during trans-cervical part of esophageal dissection can be managed through the same incision and injuries as low as carina can be successfully managed without any additional morbidity. The crux of uncomplicated repair of tracheal injuries in transhiatal esophagectomy is immediate recognition, proper exposure, interrupted suture technique, suture reinforcement and proper post-operative care.
Conflict of interest: The authors declare no conflict of interest.

\section{References}

1. Samarasam I. Esophageal cancer in India: current status and future perspectives. Int $J$ Adv Med Health Res. 2017; 4: 5-10

2. Mu J, Gao S, Xue Q, Mao Y, Wang D, Zhao J, et al. Comparison of short-term outcomes and three year survival between total minimally invasive McKeown and dual-incision esophagectomy. Thorac Cancer. 2017; 8: 80-87.

3. Harney TJ, Condon ET, Lowe D, McAnena OJ. A novel technique for repair of iatrogenic tracheal tear complicating three stage oesophagectomy. Ir J Med Sci. 2009; 178(3): 337-338.

4. LA Gorenstein, JG Abel and GA Patterson. Pericardial repair of a tracheal laceration during transhiatal esophagectomy. Ann Thorac Surg. 1992; 54(4): 784-786.

5. Foroulis CN, Simeoforidou M, Michaloudis D, Hatzitheofilou K. Pericardial patch repair of an extensive longitudinal iatrogenic rupture of the intrathoracic membranous trachea. Interact Cardiovasc Thorac Surg. 2003; 2(4): 595-597.

6. Gupta V, Gupta R, Thingnam SKS, Singh RS, Gupta AK, Kuthe $S$, et al. Major airway injury during esophagectomy: experience at a tertiary care center. J Gastrointest Surg. 2009; 13: 438-441.

7. Hulscher JB, Hofstede E, Kloek J, Obertop $\mathrm{H}$, de Haan $\mathrm{P}$, van Lanschot JJB. Injury to the major airways during subtotal esophagectomy: incidence, management, and sequelae. J Thorac Cardiovasc Surg. 2000; 120: 1093-1096.

8. Nunez JAF, Merino MCU, Escudero JA, Landeira JMV. Tracheal injury during transhiatal esophagectomy without thoracotomy: anesthesiologic management. Rev Esp Anestesiol Reanim. 1990; 37: 32-36.

9. Das AS, Dharmalingam SK, Manickam $P$, Kandasamy S. Successful management of a tracheal laceration during transhiatal esophagectomy with double lumen tube in 
situ. J Anaesth Clin Pharmacol. 2006; 22(2): 181-182.

10. Orringer MB, Marshall B, Chang AC, Lee J, Pickens A, Lau CL. Two thousand transhiatal esophagectomies. Ann Surg. 2007; 246: 363-374.

11. Suryanarayana Deo SV, Shridhar D, Shukla NK. Management of inflammatory tracheoesophageal adhesions during transhiatal esophagectomy. Surg Today. 2003; 33: 332-335.
12. Millikan KW, Pytvnia KB. Repair of tracheal defect with Gortex graft during resection of the carcinoma of the esophagus. J Surg Oncol. 1997; 66: 134-137.

13. Jha RR, Mishra S, Bhatnagar S. Rupture of left main bronchus associated with radiotherapyinduced bronchial injury and use of a doublelumen tube in oesophageal cancer surgery. Anaesth Intensive Care. 2004; 32: 104-107. 